\title{
Risks of smoking in treated and untreated older Chinese patients with isolated systolic hypertension Ji-Guang Wang ${ }^{\mathrm{a}, \mathrm{b}}$, Jan A. Staessen ${ }^{\mathrm{b}}$, Robert Fagard ${ }^{\mathrm{b}}$, Lansheng Gong ${ }^{\mathrm{c}}$ and Lisheng Liu ${ }^{a}$, for the Systolic Hypertension in China (Syst-China) Trial Collaborative Group*
}

Objectives To examine the health risks associated with smoking and blood pressure in Chinese hypertensive patients and to compare the benefit of antihypertensive drug treatment with the risk attributable to smoking.

Methods We used multiple Cox regression to correlate outcome with blood pressure and smoking status in 2284 older (aged $\geqslant 60$ years) patients enrolled in the Systolic Hypertension in China (Syst-China) Trial (systolic/diastolic blood pressure $\geqslant 160 /<95 \mathrm{mmHg}$ ).

Results Median follow-up was $\mathbf{3 . 0}$ years. After adjustment for sex, age, active antihypertensive treatment and various entry characteristics, the relative hazard rates associated with smoking more than 20 cigarettes per day were 2.04 $(P=0.04), 4.66(P<0.001)$ and $4.74(P=0.002)$ for allcause, noncardiovascular and cancer mortality, respectively. With similar adjustments applied, the relative hazard rates for total (fatal and non-fatal) stroke associated with smoking 10-20 and more than 20 cigarettes per day were $1.78(P=0.04)$ and $2.23(P=0.03)$, respectively. Furthermore, both smoking and systolic blood pressure were associated with higher risk of stroke. Compared with the overall risk in the whole group, treating 1000 patients for 5 years prevented 40 [95\% confidence interval ( $\mathrm{Cl}), 5-75]$ strokes in smokers as well as never smokers. Prognosis in never smokers and past smokers was similar. Quitting smoking had the potential to prevent $51(95 \% \mathrm{Cl},-21$ to 122$)$ strokes in untreated hypertensive patients and to prevent $45(95 \% \mathrm{Cl},-14$ to 104$)$ additional strokes in treated patients, over and above the effects of antihypertensive treatment. If, in addition to stroke, noncardiovascular mortality was also accounted for, the estimated absolute benefit of quitting smoking increased to $69(95 \% \mathrm{Cl},-18$ to 155$)$ and $97(95 \% \mathrm{Cl}, 23-171)$ events in the untreated and the treated group, respectively.

Conclusions In elderly Chinese patients, smoking was a risk factor for all-cause, non-cardiovascular and cancer mortality, as well as fatal and non-fatal stroke. The potential benefits of antihypertensive treatment and quitting smoking were approximately similar. In our view, these findings are important in terms of public health policies and health economics. J Hypertens 19:187-192 () 2001 Lippincott Williams \& Wilkins.

Journal of Hypertension 2001, 19:187-192

Keywords: smoking, hypertension, China, elderly, stroke

${ }^{a}$ Hypertension Division, Cardiovascular Institute and Fuwai Hospital, Chinese Academy of Medical Sciences and Peking Union Medical College, Beijing, People's Republic of China, b Study Coordinating Centre, Hypertension and Cardiovascular Rehabilitation Unit, Department of Molecular and Cardiovascular Research, University of Leuven, Leuven, Belgium and ${ }^{\mathrm{C}}$ Shanghai Hypertension Institute, Shanghai Second Medical University, Shanghai, People's Republic of China.

*A complete list of members of the Syst-China Collaborative Group appears in reference [13].

Sponsorship: Syst-China was financially supported by the State Planning Commission of the People's Republic of China. The present analysis was facilitated through a fellowship granted by Bayer AG, Wuppertal, Germany, to J.-G.W.

Correspondence and requests for reprints to Jan A. Staessen,

Studiecoördinatiecentrum, Laboratorium Hypertensie, Gebouw Onderwijs en Navorsing, Campus Gasthuisberg, Herestraat 49, B-3000 Leuven, Belgium. Tel: +32163471 04; fax: +3216347106;

e-mail: jan.staessen@med.kuleuven.ac.be

Received 7 July 2000 Revised 24 October 2000 Accepted 25 October 2000

\section{Introduction}

Among the 1.1 billion smokers worldwide, 330 million live in China [1]. In 1990, the annual sales volume of cigarettes in China was 16 billion and had doubled since 1981 [1]. In elderly Chinese men and women, the percentage of smokers amounts to $35 \%$ [1,2]. The prevalence of hypertension in elderly Chinese exceeds $30 \%$ [3]. Due to increasing longevity and family planning policies, people aged over 60 years represent more than $10 \%$ of the 1.2 billion Chinese. Elderly smokers are often exposed to other risk factors, such as hypertension, which may further increase the health risk [4]. Previous studies in Chinese consistently demonstrated that smoking was associated with the risk of cancer and non-cardiovascular mortality [5-10]. However, unlike findings in Western populations [11], the Chinese prospective studies failed to show a significant relationship between smoking and stroke, which is the most frequent cardiovascular disorder in China $[5,7,12]$. 
The present study builds on the results of the Systolic Hypertension in China (Syst-China) Trial, which included 737 current smokers among the 2284 enrolled patients in whom information on smoking was recorded $[13,14]$. We examined the health risks associated with smoking in treated and untreated Syst-China patients and compared the benefit of antihypertensive treatment with the risk attributable to smoking.

\section{Subjects and methods}

The Chinese Ministry of Health approved the SystChina trial. Its design and the definition and validation of endpoints are described in detail elsewhere $[13,15,16]$.

Eligible patients consented to be enrolled and were at least 60 years old. On single-blind placebo during the run-in phase, their sitting systolic blood pressure had to range from 160 to $219 \mathrm{mmHg}$, with diastolic blood pressure below $95 \mathrm{mmHg}$. The sitting blood pressure determining eligibility was the average of six readings, two at three baseline visits 1 month apart. Patients whose serum creatinine concentration exceeded $2 \mathrm{mg} /$ $\mathrm{dl}$ and patients with severe concomitant cardiovascular or noncardiovascular disorders were excluded $[13,15,16]$.

Eligible patients were assigned active antihypertensive treatment or placebo. Active antihypertensive treatment was initiated with nitrendipine $(10-40 \mathrm{mg} / \mathrm{day})$, with the possible addition of captopril $(12.5-50 \mathrm{mg} /$ day), hydrochlorothiazide (12.5-50 mg/day), or both drugs. The study drugs were stepwise titrated and combined to reduce the sitting systolic blood pressure by $20 \mathrm{mmHg}$ or more to less than $150 \mathrm{mmHg}$ $[13,15,16]$. Placebos were used in the same way as the active drugs. During follow-up, patients were examined at monthly intervals. Patients who withdrew from the study medication remained in open follow-up. Patients without any report within the year before the trial ended were classified as lost to follow-up, but were included in the analysis up to the most recent evaluation of their health status $[13,15,16]$.

At the screening visit, patients were questioned on smoking habits and alcohol intake. Never smokers were patients who had never used tobacco in their lifetime. We classified patients who had quit smoking before entry as past smokers $[13,15,16]$. Diabetes mellitus at baseline was defined according to the criteria of the World Health Organization [17]. The diagnosis required the presence of a history of diabetes reported by the clinical investigator, a fasting blood glucose level of $7.8 \mathrm{mmol} / \mathrm{l}$ or more, or the use of anti-diabetic medication.

Stroke was defined as a neurological deficit with symptoms continuing for more than $24 \mathrm{~h}$ or leading to death with no apparent cause other than vascular. Cardiovascular mortality included sudden death and deaths due to stroke, cardiac endpoints, or other cardiovascular disease. Noncardiovascular mortality included all other deaths except those in whom the cause of death could not be identified.

The statistical analysis was performed with SAS software (SAS Institute Inc, Cary, North Carolina, USA), version 6.12, using two-sided tests. Means and proportions were contrasted by the $t$-test and the chi-squared statistic, respectively. The incidence rates by smoking status at entry were calculated with standardization for sex and age by the direct method [18]. We used multiple Cox regression to study the association between outcome and predictors [19]. From one overall model adjusted for confounders, we computed the risk of an adverse outcome in four subgroups according to antihypertensive treatment group and smoking status relative to the overall risk in the whole sample, using the deviation-from-means coding approach [20]. Absolute risks and 95\% confidence intervals (CI) were calculated from the rates in the whole sample and in the four subgroups and from the standard errors of the differences between these rates.

\section{Results}

Of the 2394 patients enrolled in the trial, $110(4.6 \%)$ were excluded because their smoking status had not been recorded at entry, leaving 2284 patients for analysis (Table 1). At baseline, 1278 patients (55.9\%) had never smoked, 269 (11.8\%) were past smokers, and 737 $(32.3 \%)$ were current smokers. Among current smokers, $241(32.7 \%), 368(49.9 \%)$ and $128(17.4 \%)$ smoked 1-9, 10-20, and more than 20 cigarettes per day, respectively. The percentages of past and current smokers were higher in 1492 men than in 792 women $(15.4$ versus $5.1 \%$ and 42.0 versus $14.0 \%$, respectively; $P<$ 0.001). Compared with never smokers, past and current smokers more frequently drank alcohol and more past smokers had previously experienced cardiovascular complications $(P<0.001)$. The differences in the other baseline characteristics across smoking categories were small or non-significant.

Median follow-up was 3.0 years (range 1-94 months). The total number of patient-years of follow-up was 6414. In never smokers, total, noncardiovascular and cancer mortality amounted to 64, 28 and 13 deaths, and 72 fatal and non-fatal cardiovascular events and 44 strokes occurred. In past smokers, the corresponding numbers were $16,7,6,72$ and 44 , and in current smokers 55, 26, 18, 65 and 46. After adjustment for sex and age (Fig. 1), smoking was associated with higher mortality from noncardiovascular diseases and cancer and with higher cardiovascular event rates. This was 
Table 1 Characteristics of patients according to smoking status at baseline

\begin{tabular}{|c|c|c|c|c|}
\hline Characteristic & Never smokers & Ever smokers & Past smokers & Current smokers \\
\hline Men $(n=1492)$ & 637 & 855 & 229 & 626 \\
\hline Age (years) & $67 \pm 5$ & $67 \pm 5$ & $68 \pm 5^{*}$ & $66 \pm 5^{*}$ \\
\hline Body mass index $\left(\mathrm{kg} / \mathrm{m}^{2}\right)$ & $23.8 \pm 3.1$ & $23.7 \pm 3.3$ & $24.6 \pm 3.6^{* *}$ & $23.4 \pm 3.2^{*}$ \\
\hline Systolic blood pressure $(\mathrm{mmHg})$ & $170 \pm 11$ & $171 \pm 11$ & $171 \pm 11$ & $170 \pm 11$ \\
\hline Diastolic blood pressure $(\mathrm{mmHg})$ & $88 \pm 6$ & $86 \pm 6^{* * *}$ & $87 \pm 6$ & $86 \pm 7^{* * *}$ \\
\hline Pulse rate (beats/min) & $77 \pm 10$ & $76 \pm 9$ & $75 \pm 10$ & $76 \pm 9$ \\
\hline Serum total cholesterol $(\mathrm{mmol} / \mathrm{l})$ & $5.0 \pm 1.2$ & $5.0 \pm 1.2$ & $5.1 \pm 1.3$ & $4.9 \pm 1.1$ \\
\hline Alcohol intake, $n(\%)$ & $235(36.9)$ & $503(58.8)^{* * *}$ & $113(49.3)^{* * *}$ & $390(62.3)^{* * *}$ \\
\hline Previous CV complications & $69(10.8)$ & $108(12.6)$ & $45(19.7)^{* * *}$ & $63(10.1)$ \\
\hline Diabetic patients & $23(3.6)$ & $31(3.6)$ & $10(4.4)$ & 21 (3.4) \\
\hline Women $(n=792)$ & 641 & 151 & 40 & 111 \\
\hline Age (years) & $66 \pm 5$ & $68 \pm 6^{* * *}$ & $70 \pm 6^{* * *}$ & $67 \pm 6^{* *}$ \\
\hline Body mass index $\left(\mathrm{kg} / \mathrm{m}^{2}\right)$ & $24.5 \pm 3.8$ & $23.4 \pm 3.8^{* * *}$ & $23.3 \pm 3.7$ & $23.4 \pm 3.8^{* *}$ \\
\hline Systolic blood pressure $(\mathrm{mmHg})$ & $171 \pm 11$ & $172 \pm 13$ & $176 \pm 14^{*}$ & $171 \pm 13$ \\
\hline Diastolic blood pressure $(\mathrm{mmHg})$ & $85 \pm 7$ & $84 \pm 8^{*}$ & $83 \pm 8^{*}$ & $84 \pm 9$ \\
\hline Pulse rate (beats/min) & $77 \pm 9$ & $76 \pm 10$ & $76 \pm 10$ & $76 \pm 11$ \\
\hline Serum total cholesterol $(\mathrm{mmol} / \mathrm{l})$ & $5.3 \pm 1.2$ & $5.5 \pm 1.3$ & $5.6 \pm 1.9$ & $5.5 \pm 1.0$ \\
\hline Alcohol intake, $n(\%)$ & $50(7.8)$ & $38(25.2)^{* * *}$ & $9(22.5)^{* *}$ & $29(26.1)^{* * *}$ \\
\hline Previous CV complications & $63(9.8)$ & 20 (13.3) & $7(17.5)$ & $13(11.7)$ \\
\hline Diabetic patients & $34(5.3)$ & $6(4.0)$ & $2(5.0)$ & $4(3.6)$ \\
\hline All subjects $(n=2284)$ & 1278 & 1006 & 269 & 737 \\
\hline Age (years) & $66 \pm 6$ & $67 \pm 5^{*}$ & $68 \pm 5^{* * *}$ & $66 \pm 5$ \\
\hline Body mass index $\left(\mathrm{kg} / \mathrm{m}^{2}\right)$ & $24.2 \pm 3.5$ & $23.7 \pm 3.4^{* * *}$ & $24.5 \pm 3.6$ & $23.4 \pm 3.3^{* * *}$ \\
\hline Systolic blood pressure $(\mathrm{mmHg})$ & $170 \pm 11$ & $171 \pm 11$ & $172 \pm 12$ & $170 \pm 11$ \\
\hline Diastolic blood pressure (mmHg) & $86 \pm 7$ & $86 \pm 7$ & $86 \pm 6$ & $86 \pm 7^{*}$ \\
\hline Pulse rate (beats/min) & $77 \pm 9$ & $76 \pm 9^{*}$ & $76 \pm 10^{*}$ & $76 \pm 9$ \\
\hline Serum total cholesterol $(\mathrm{mmol} / \mathrm{l})$ & $5.2 \pm 1.2$ & $5.0 \pm 1.2^{*}$ & $5.1 \pm 1.5$ & $5.0 \pm 1.1^{* *}$ \\
\hline Alcohol intake, $n(\%)$ & $285(22.3)$ & $541(53.8)^{* * *}$ & $122(45.4)^{* * *}$ & $419(56.9)^{* * *}$ \\
\hline Previous CV complications & $132(10.3)$ & $128(12.7)$ & $52(19.4)^{* * *}$ & $76(10.3)$ \\
\hline Diabetic patients & $57(4.5)$ & $37(3.7)$ & $12(4.5)$ & $25(3.4)$ \\
\hline
\end{tabular}

Values are means $\pm S D$, unless otherwise indicated. CV, cardiovascular. Significance of difference with never smokers: ${ }^{*} P<0.05 ;{ }^{* *} P<0.01$; $^{* * *} P<0.001$.

confirmed after further adjustment for active antihypertensive treatment and various entry characteristics, such as systolic and diastolic blood pressure, body mass index, serum total cholesterol, alcohol intake, previous cardiovascular complications and diabetes (Table 2). The relative hazard rates associated with smoking more than 20 cigarettes per day were $2.04(P=0.04), 4.66$ $(P<0.001)$ and $4.74(P=0.002)$ for all-cause, noncardiovascular and cancer mortality, respectively. The cancers originated from the oesophagus and stomach in seven cases, from the colon in four, from the liver and pancreas in five, from the lung in 10 and from other sites in 11 patients. The increased cancer mortality in current smokers was mainly due to lung cancer (relative hazard versus never smokers: 10.2; 95\% CI, 1.2-85.9, $P=0.03)$.

Smoking was also significantly associated with higher risk of fatal and non-fatal stroke (Fig. 1 and Table 2). After cumulative adjustments for sex, age and significant covariates, the relative hazard rates associated with smoking $10-20$ and more than 20 cigarettes per day were $1.78(P=0.04)$ and $2.23(P=0.03)$, respectively. In moderate and heavy smokers combined, compared with never smokers, the adjusted relative hazard rate for fatal stroke was 2.66 (95\% CI, 1.06-6.69, $P=0.04)$.
After similar adjustments as before, cardiovascular mortality and the incidence of all fatal and non-fatal cardiovascular endpoints rose with increasing systolic blood pressure at baseline in never smokers, but not in smokers. The $P$-values for the interaction terms were 0.02 and 0.05 , respectively. The relative hazard rates associated with a $10 \mathrm{mmHg}$ higher systolic blood pressure at baseline were 1.37 (95\% CI, 1.03-1.81, $P=$ $0.03)$ and $1.40(1.15-1.71, P<0.001)$ in never smokers, but only $0.80(0.57-1.14, P=0.21)$ and $1.02(0.82-$ $1.28, P=0.86)$ in smokers.

Serum total cholesterol at baseline was higher in women than in men $[5.36 \pm 1.22$ (SD) versus $4.96 \pm 1.20 \mathrm{mmol} / \mathrm{l} ; \quad P<0.001]$. After adjustment for sex, age and other potential confounders, serum total cholesterol was not associated with any endpoint; the $P$-values ranged from 0.17 for cancer mortality to 0.93 for cardiovascular mortality. There was also no interaction between smoking and serum total cholesterol for any endpoint $(P>0.40)$.

The relative and absolute risks of stroke, noncardiovascular mortality, and both events combined were estimated in subgroups according to antihypertensive treatment group and smoking status (Fig. 2). Both in 
Fig. 1

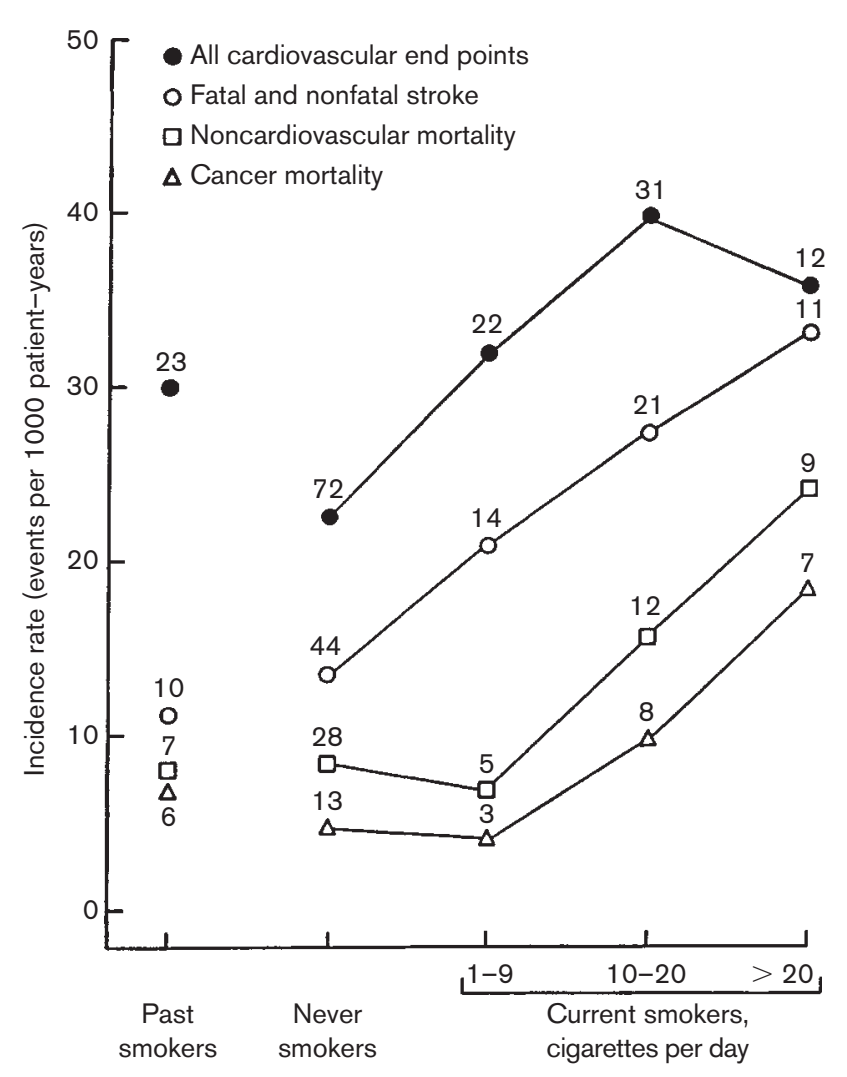

Sex- and age-adjusted event rates by smoking status.

relative and absolute terms, the risks were consistently higher in untreated than in treated patients and in current smokers than in never smokers. The treated never smokers had the lowest risk, whereas the untreated smokers had the highest risk. Treated smokers and untreated never smokers were exposed to similar and intermediate risks.

\section{Discussion}

Stroke is the most frequent cardiovascular complications of hypertension in China [21,22]. A major new finding of our prospective study was that, in older Chinese hypertensive patients, smoking was significantly associated with stroke. Compared with the overall risk in the whole group, treating 1000 patients for 5 years prevented 40 (95\% CI, 5-75) strokes in smokers and never smokers alike. However, if the risk of smoking would be reversible, as suggested by the similar risks in never smokers and past smokers, quitting smoking would have the potential to prevent 51 (95\% CI, -21 to 122) strokes in untreated hypertensive patients and to prevent 45 (95\% CI, -14 to 104) additional strokes in treated patients, over and above the effects of antihypertensive treatment. If, in

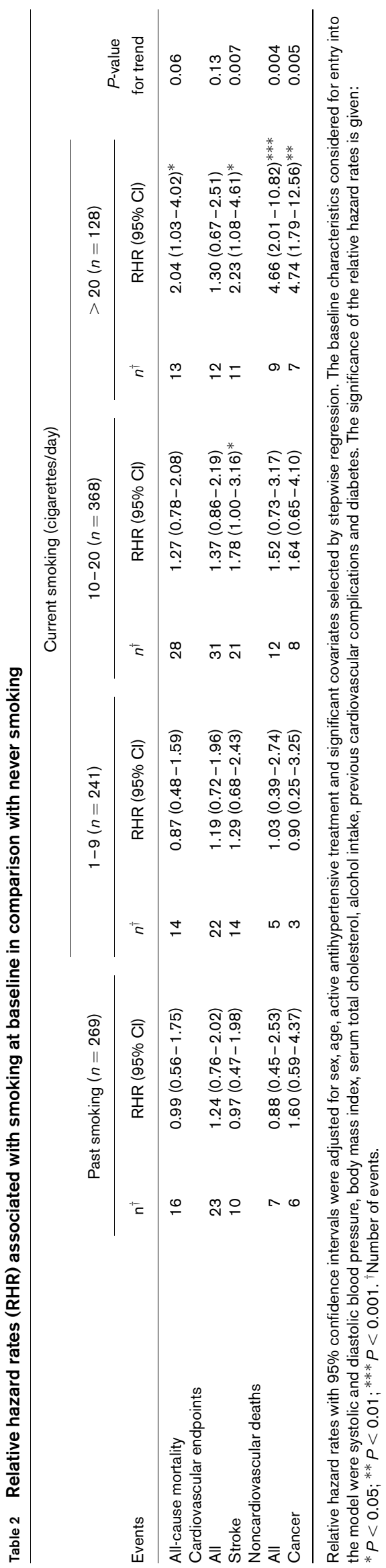




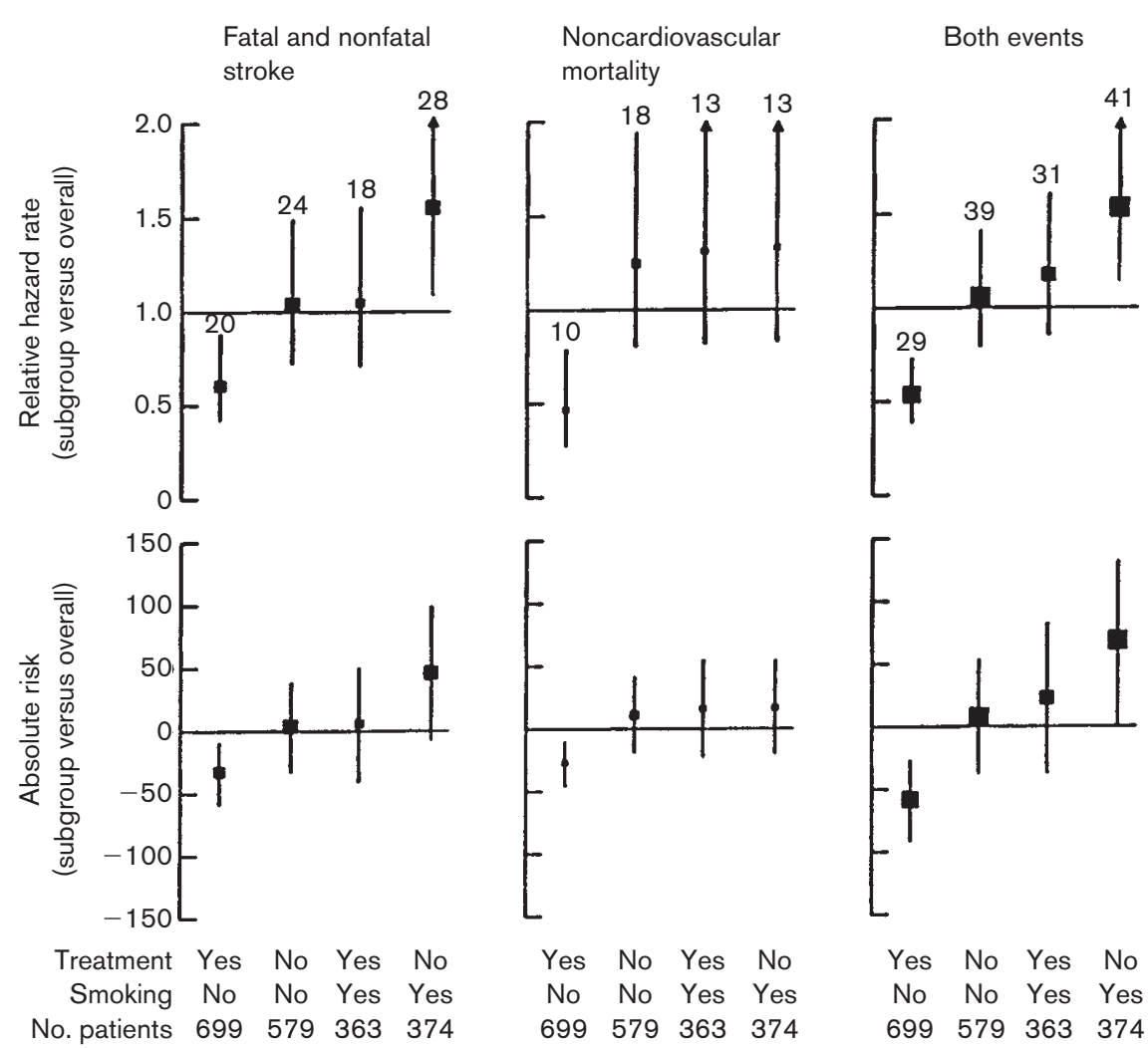

Relative (upper) and absolute (lower) risk associated with antihypertensive treatment and smoking status adjusted for sex, age, systolic and diastolic blood pressure at entry, previous cardiovascular complications, alcohol intake and diabetes. Solid squares represent risks of stroke, noncardiovascular death, and both events in four subgroups in comparison with the overall risk in the whole study population. Sizes of the squares are proportional to number of events in each subgroup. Because only the first event was accounted for, numbers of strokes and non-cardiovascular deaths do not add up. Vertical lines denote $95 \%$ confidence intervals. Absolute risk was expressed as the number of events per 1000 patients followed for 5 years.

addition to stroke, non-cardiovascular mortality was also accounted for, the estimated absolute benefit of quitting smoking increased to 69 (95\% CI, -18 to 155$)$ and 97 (95\% CI, 23-171) events in the untreated and the treated group, respectively. Thus, the potential benefits of antihypertensive treatment and quitting smoking are approximately similar. In our view, these findings are important in terms of health economics and public health policies.

In Western populations, the role of smoking as a risk factor for stroke is well established in cohort and casecontrol studies [11], whereas in three Chinese prospective studies it remained equivocal $[5-7,12]$. In none of these studies was there a significant association between smoking status and stroke incidence although statistical power was sufficiently large. Indeed, the sample size ranged from 1696 [7] to 18244 [6,12], the follow-up duration from $6[6,12]$ to 19 years [7], and the number of fatal strokes from 41 [7] to $245[6,12]$. The negative findings in the three large cohort studies could be explained by inaccurate diagnosis or under-reporting. Indeed, the diagnosis of stroke was usually made on the basis of death certificates, hospital records, municipal vital statistics, or interrogating patients or their family. In contrast, in the Syst-China trial, stroke was the primary endpoint validated by blinded and independent investigators [13]. Moreover, the SystChina patients were followed up at monthly intervals, which probably decreased the possibility of underreporting of events. In addition, previous prospective studies were conducted in the general population [5$7,12]$, whereas the subjects in the present study were older hypertensive patients. Old age and hypertension may have further increased the risk of stroke associated with smoking [4], thus making it possible to detect a significant association.

Other Chinese studies with a cross-sectional $[8,9]$ or case-control [10] design showed a weak but significant association between smoking status and stroke incidence. According to the 1986 Chinese National Stroke 
Survey and the 1979-80 Chinese National Hypertension Survey, a $10 \%$ increase in the prevalence of cigarette smoking was associated with a $19 \%$ higher stroke mortality [8]. In a large retrospective study of 1 million deaths from urban and rural areas of China, stroke mortality was slightly more common among smokers (risk ratio 1.17) [10]. In a cross-sectional survey of 2600 Chinese residents aged 65 years or older living in Taiwan, the odds ratios associated with heavy smoking were 1.71 (95\% CI, 1.04-2.80) for all strokes combined and 1.72 (95\% CI, 1.00-2.96) for cerebral infarction [9].

The significant interaction between smoking and systolic blood pressure could be a chance finding due to the small number of events when two factors were considered simultaneously. However, it is also possible that the lack of association between the cardiovascular event rate and systolic blood pressure in elderly smokers is due to selection, because fewer smokers than never smokers left until age 60 years [4].

A positive association between serum total cholesterol and coronary events has been observed in middle-aged hypertensive patients [23]. However, in studies in the elderly, total serum cholesterol is often not positively associated with cardiovascular and noncardiovascular mortality [18]. In Asian populations, the association between serum total cholesterol and stroke is weak [24] and there is even a tendency of an inverse correlation with haemorrhagic stroke [24,25]. In addition, the null findings in the present study could be due to the relatively low level of cholesterol and the low rate of coronary events in Chinese [21,22].

In conclusion, smoking is not only associated with mortality from noncardiovascular disease and cancer, but also entails a higher stroke risk in older Chinese patients with isolated systolic hypertension. Approximately 1.6 million incident strokes occur each year in China [26], a developing nation in which health care resources are still scarce, especially in rural areas. Voluntary smoking cessation, regulation of tobacco advertising and banning smoking from public areas [27] should be reinforced as high priority measures for the primary prevention of stroke in China.

\section{References}

1 Yang G, Fan L, Tan J, Qi G, Zhang Y, Samet JM, et al. Smoking in China. Findings of the 1996 National Prevalence Survey. JAMA 1999; 282: 1247-1253.

2 Koplan JP, Feng W, Chen CHC, Zheng P, Harris JR. Cigarette smoking in China. Prevalence, characteristics, and attitudes in Minhang District. JAMA 1995; 274:1232-1234.

3 Wu X, Duan X, Gu D, Hao J, Tao S, Fan D. Prevalence of hypertension and its trend in Chinese populations. Int J Cardiol 1995; 52:39-44.

4 Lowe LP, Greenland P, Ruth KJ, Dyer AR, Stamler R, Stamler J. Impact of major cardiovascular disease risk factors, particularly in combination, on 22 year mortality in women and men. Arch Intern Med 1999; 158:2007-2014.
5 Chen ZM, Xu Z, Collins R, Li WX, Peto R. Early health effects of the emerging tobacco epidemic in China. JAMA 1997; 278:1500-1504.

6 Yuan JM, Ross RK, Wang XL, Gao YT, Henderson BE, Yu MC. Morbidity and mortality in relation to cigarette smoking in Shanghai, China. A prospective male cohort study. JAMA 1996; 275:1646-1650.

7 Lam TH, He Y, Li LS, He SF, Liang BQ. Mortality attributable to cigarette smoking in China. JAMA 1997; 278:1505-1508.

8 He J, Klag MJ, Wu Z, Whelton PK. Stroke in the People's Republic of China. I. Geographic variations in incidence and risk factors. Stroke 1995; 26:2222-2227.

9 Lee TK, Huang ZS, Ng SK, Chan KWA, Wang YS, Liu HW, et al. Impact of alcohol consumption and cigarette smoking on stroke among the elderly in Taiwan. Stroke 1995; 26:790-794.

10 Liu BQ, Peto R, Chen ZM, Boreham J, Wu YP, Li JY, et al. Emerging tobacco hazards in China: 1. Retrospective proportional mortality study of one million deaths. Br Med J 1999; 317:1411-1422.

11 Hankey GJ. Smoking and risk of stroke. J Cardiovasc Risk 1999; 6: 207-211.

12 Ross RK, Yuan JM, Henderson BE, Park J, Gao YT, Yu MC. Prospective evaluation of dietary and other predictors of fatal stroke in Shanghai, China. Circulation 1997; 96:50-55.

13 Liu L, Wang JG, Gong L, Liu G, Staessen JA, for the Systolic Hypertension in China (Syst-China) Collaborative Group. Comparison of active treatment and placebo for older patients with isolated systolic hypertension. $J$ Hypertens 1998; 16:1823-1829.

14 Wang JG, Staessen JA, Gong L, Liu L, for the Systolic Hypertension in China Investigators. Chinese trial on isolated systolic hypertension in the elderly. Arch Intern Med 1999; 160:211-220.

15 Wang JG, Liu G, Wang X, Zhang S, Sun M, Pan X, et al. Long-term blood pressure control in older Chinese patients with isolated systolic hypertension: a progress report on the Syst-China trial. J Hum Hypertens 1996; 10:735-742.

16 Collaborative Group Coordinating Center. Systolic hypertension in the elderly: Chinese trial (Syst-China) - Interim report. Chin J Cardiol 1992; 20:270-275.

17 Report of a WHO Study Group. Prevention of diabetes mellitus. Geneva Switzerland: World Health Organization; 1994.

18 Staessen J, Amery A, Birkenhäger W, Bulpitt C, Clement D, de Leeuw P, et al. Is a high serum cholesterol level associated with longer survival in elderly hypertensives? J Hypertens 1990; 8:755-761.

19 Cox DR. Regression models and life tables. JR Stat Soc B 1972; 34 $187-220$.

20 Hosmer DW Jr, Lemeshow S. Applied logistic regression. New York, New York, USA: John Wiley \& Sons; 1989.

21 Tao SC, Zhang XG, Wang SY, Yu XH, Wu XG, Lu CQ. Incidence of myocardial infarction and stroke in the Capital iron and steel complex region of Beijing, China. Magnesium 1982; 1:144-150.

22 Shi F, Hart RG, Sherman DG, Tegeler CH. Stroke in the People's Republic of China. Stroke 1989; 20:1581-1585.

23 The IPPPSH Collaborative Group. Cardiovascular risk and risk factors in a randomized trial of treatment based on the beta-blocker oxprenolol: the International Primary Prevention Study in Hypertension (IPPPSH). J Hypertens $1985 ; 3: 379-392$.

24 Eastern Stroke and Coronary Heart Disease Collaborative Group. Blood pressure, cholesterol, and stroke in eastern Asia. Lancet 1998; 352: $1801-1807$.

25 Yano K, Reed DM, MacLean CJ. Serum cholesterol and hemorrhagic stroke in the Honolulu Heart Program. Stroke 1989; 20:1460-1465.

26 Li SC, Schoenberg BS, Wang CC, Cheng XM, Bolis CL, Wang KJ. Cerebrovascular disease in the People's Republic of China: epidemiologic and clinical features. Neurology 1985; 35:1708-1713.

27 Tomlinson R. Shanghai bans public smoking. B M J 1994; 309: 1460-1460. 